\section{Knowledge, attitudes and eye care seeking practices regarding trachoma in Central Division of Kajiado County, Kenya}

\author{
Arthur S. Ng'etich, ${ }^{1}$ Claudio Owino, ${ }^{2}$ \\ Ahmad Juma, ${ }^{1}$ Kevin N. Khisa ${ }^{1}$ \\ ${ }^{1}$ Department of Epidemiology and \\ Biostatistics, School of Public Health and \\ 2Department of Surgery and \\ Anesthesiology, School of Medicine, Moi \\ University, Kenya
}

\begin{abstract}
Trachoma is responsible for the visual impairment of about two million people worldwide, out of which a majority are irreversibly blind. The study aimed to assess the level of knowledge, attitudes and eye care seeking practices of the community regarding the available trachoma eye care services. Community members aged eighteen and over participated. Descriptive cross-sectional study design was adapted. Data analysis was performed using Stata. The level of awareness of trachoma disease in the study area was high (95\%). About three quarters (71\%) of the respondents were knowledgeable of the available trachoma eye care services. A majority had a positive attitude towards the available services and expressed need for the services. Public health facilities were mostly preferred. The study concluded that the community was knowledgeable of the available trachoma eye care services and had a positive attitude towards the services. Concerned stakeholders should reinforce the positive attitudes through community based educational programs and improve eye care seeking practices through community participation in education and outreach services.
\end{abstract}

\section{Introduction}

Neglected tropical diseases (NTDs) are one of the key areas of concern for our society. These communicable diseases affect an estimated one billion people globally, primarily poor populations living in tropical and subtropical climates, with children being the most vulnerable to infection. ${ }^{1}$ Trachoma is one of the neglected tropical infectious diseases and a leading cause of preventable blindness found among the poorest of the communities in the world. Globally, more than two million people are either blind or suffer from a very painful disability because of trachoma. A further 4.6 million have got to the stage of the disease where they are in need of surgery to stop them going blind. Trachoma blinds four people every hour which translates to every fifteen minutes someone going blind from the disease. ${ }^{2}$

Trachoma begins in childhood with an acute infection of Chlamydia trachomatis, and progresses over the years with repeated infections. Scarring and irritation caused by chronic inflammation of the conjunctiva (the inner eyelid) cause the eyelid to shrink and the eyelashes to turn inward, scraping the cornea. If left untreated, this condition, trichiasis, may lead to corneal opacity and eventual blindness. ${ }^{3}$

Trachoma disease is exacerbated by various community-based practices and environmental factors. Some of the risks factors predisposing communities to the disease include; women who care for children putting them frequently in close contact with children who are the sole reservoirs of the disease causing bacteria, water scarcity in arid and semi-arid areas where trachoma disease is mostly endemic reducing use of water for personal hygiene purposes such as facial cleanliness, utilization of sanitary facilities and indiscriminate defecation in bushes and proximity of livestock corrals to the households resulting in high fly population and the household general sanitary conditions such as poor dusting of earthen floored households acting as formites for transmission of the disease causing bacteria to the human eye. These predisposing factors have an impact on the prevalence and burden of trachoma disease in trachoma endemic regions.

In an effort to eliminate trachoma in Kenya, the Ministry of Health with support from Africa Medical Research Foundation (AMREF), initiated the Trachoma Integrated Project in the Kajiado District in 2007. The goal was to reduce the overall prevalence of active trachoma among children aged 1-9 years from $23 \%$ to less than $10 \%$ by the year 2010 but according to a recent survey carried out in Kajiado County by AMREF in the year 2013, the prevalence of active trachoma was found to be $17.4 \%$. Therefore, indicating the need for enhanced interventions to ensure effective control and treatment of the eye disease to bring down the prevalence of the disease. ${ }^{4}$ Several studies have been done regarding the risk factors of trachoma disease in different parts of the world where trachoma is endemic. A lot more has also been documented regarding the interventions put in place to prevent and control the eye condition in communities affected by the disease. However, despite the documentation of all these information, the prevalence of trachoma in many areas including Kajiado County remains above the World
Correspondence: Arthur Saitabau Ng'etich, Department of Epidemiology and Biostatistics, Moi University, P.0. Box 7470-30100, EldoretKenya.

Tel.: +25.4710.890.400.

E-mail: arthursaitabau@yahoo.com

Key words: Trachoma; eye care services; knowledge; attitudes; eye care seeking practices.

Acknowledgements: the authors would like to thank the Medical Superintendent-Kajiado Sub County Hospital, Africa Medical and Research Foundation Trachoma Integrated Project Manager and Project Officer - Mr. Dikir and Mr. Ochieng' respectively, research assistants and the community members in Kajiado County who participated this research.

Contributions: ASN, research idea conception, study designing, data collection, analysis, and interpretation of data, drafting and revising the manuscript and final review and approval of final version of manuscript for publication; $\mathrm{CO}$, study design, interpretation of manuscript, revising manuscript and final approval of version to be published; AJ, collection of data and data analysis, revising of manuscript and review and approval of the final version for publication; KNK, data analysis, checking references, revising of manuscript and approval of the final version for publication.

Conflict of interest: the authors declare no potential conflict of interest.

Dedication: the article is dedicated to community members of Kajiado County.

Received for publication: 4 February 2016

Revision received: 1 March 2016.

Accepted for publication: 2 March 2016.

This work is licensed under a Creative Commons Attribution NonCommercial 3.0 License (CC BYNC 3.0).

(C) Copyright A.S. Ng'etich et al., 2015

Licensee PAGEPress, Italy

Journal of Public Health in Africa 2015; 6:531 doi:10.4081/jphia.2015.531

Health Organization threshold (Trachomatous Follicular <10\%) for the disease to be no longer considered a public health concern in a given population. Therefore, this study tries to establish whether the communities' level of knowledge, attitudes and eye care seeking practices towards the available trachoma eye care services affects the use of these services provided through the interventions currently in place in the region.

The Ministry of Health and other humanitarian organizations in Kajiado County have put in their concerted efforts to reduce the burden of this debilitating eye disease through various trachoma interventions. Despite the 
availability of these eye care services, no data was found regarding the communities' level of knowledge, attitudes and eye care seeking practices towards these services. Researchers have limited the focus of their studies to the risk factors of trachoma disease and the interventions put in place towards prevention and control of the disease but there is inadequate literature as to whether the aforementioned factors affect utilization of the available trachoma eye care services and hence, the continued high prevalence of the disease in the region. Therefore, this study intends to provide a broad understanding of community's level of knowledge, their perception and attitudes and their eye care seeking practices towards the available eye care services. Recommendations from this study will enable the concerned stakeholders improve the currently existing interventions towards prevention and control of the blinding disease and in effect bring down the prevalence towards elimination of the disease in the region. The three aspects of knowledge of the available eye care services, attitudes towards the services and eye care seeking practices of the community play a significant role in determining use of eye care services. In a study of knowledge, attitudes and practices regarding eye surgery in parts of India, it was found that poor knowledge regarding eye diseases were reported and respondents were unaware of the possibilities to get their sight restored through surgical operations. ${ }^{5}$ According to Ashaye, Ajuwon and Adeoti (2006), many people especially in the developing regions are still becoming blind due to barriers such as beliefs and attitudes.
They found that beliefs and attitudes of the predominantly rural population are still major barriers to utilization of eye care services in Nigeria. ${ }^{6}$ Similarly, the communities in Kajiado County which is relatively remote are likely to observe certain beliefs and attitudes towards the available trachoma eye care services.

\section{Materials and Methods}

The study was conducted in Central division of Kajiado County. Central division has thirteen locations: Elangata-Wuas, Enkaroni, Enkorika, Ildamat, Kikuro, Kilonito, Loodokilani, Nkoile, Olibelibel, Olontulungum, Sajiloni, Township and Torosei. The target population of the study comprised of all the community members aged eighteen and over. A cross-sectional study design was adapted.

\section{Sample size and sampling tech- niques}

A sample size of three hundred and twenty one respondents was determined in accordance with the Fisher's Formula. ${ }^{7}$ The study respondents in Central division were selected by stratified sampling by locations and simple random sampling was used to select the households in each location until the desired sample size was reached. Convenient sampling was applied in the selection of respondents within households depending on their availability and willingness to participate in the study.

\section{Data collection instrument}

Respondents were interviewed through an interviewer-administered questionnaire. This tool obtained information on respondents' knowledge of the trachoma eye care services available to them, attitudes towards these services, and their eye-care seeking practices in relation to the available eye care services.

\section{Study procedure}

All the thirteen locations within Central division of Kajiado County were selected. The sample size was proportionately allocated to the thirteen strata. Microsoft Excel was used to generate random numbers adding up to the required sample size. Unique identifiers (i.e. the name of household heads) used during the 2009 National Population and Housing Census were used to generate a list of all the households in each of the locations in Central Division. If a random number matched a household's unique identifier, that household was automatically added to the list of selected households. The list of selected households in their respective sub-locations and villages was used to interview the study participants. Only the household heads were interviewed but in their absence, a household member aged eighteen years or older was interviewed instead.

\section{Ethical considerations}

An approval from the institutional research and ethics committee was obtained prior to commencement of the study. Informed consent

Table 1. Communities' level of knowledge of available trachoma eye care services.

\begin{tabular}{|c|c|c|}
\hline Variables, levels & Sample size & No. $(\%)$ \\
\hline $\begin{array}{l}\text { Awareness of trachoma disease } \\
\text { Yes vs. no }\end{array}$ & 321 & $305(95)$ \\
\hline $\begin{array}{l}\text { Knowledge on any facility or organization in the region that offers trachoma eye care services to the community } \\
\text { Yes us. no }\end{array}$ & 320 & $159(50)$ \\
\hline $\begin{array}{l}\text { Knowledge on specific health facilities or NGOs offering trachoma eye care services } \\
\text { Public } \\
\text { NGOs and other organizations } \\
\text { Private } \\
\text { Public and private } \\
\text { Public and NGOs } \\
\text { Private and NGOs } \\
\text { Private, public and NGO } \\
\text { Chemists }\end{array}$ & 159 & $\begin{array}{l}66(42) \\
14(9) \\
8(5) \\
24(15) \\
26(16) \\
12(8) \\
6(4) \\
3(1)\end{array}$ \\
\hline $\begin{array}{l}\text { Knowledge on any trachoma eye care services provided by public or private health facilities or NGO's } \\
\text { Yes us. no }\end{array}$ & 312 & $224(71)$ \\
\hline $\begin{array}{l}\text { Knowledge on the specific trachoma eye care services offered by public or private health facilities or NGO's } \\
\text { Eye examination } \\
\text { Eye surgery } \\
\text { Antibiotic treatment } \\
\text { Antibiotic treatment and eye surgery } \\
\text { Antibiotic treatment and eye examination }\end{array}$ & 224 & $\begin{array}{c}4(1.8) \\
2(0.9) \\
186(83) \\
28(12.5) \\
4(1.8)\end{array}$ \\
\hline
\end{tabular}

NGO, non-governmental organizations. 
was sought from respondents involved in the study with the aim of the study explained in detail and all the information was to be treated with utmost confidentiality.

\section{Data analysis}

Data was then entered in computerized Ms Access database and was later exported to Stata version 12 for analysis. Categorical variables were summarized as frequencies and corresponding percentages. The test for normality was done using the Shapiro-Wilks test for normality. The association between categorical variables was assessed using the Pearson's Chi Square test at 95\% confidence interval.

\section{Results}

A total of 321 participants were included in the study. Of this number, 64 (20\%) were aged 18-24 years, 219 (68\%) were aged $25-59$ years. The rest were aged 60 years and over. The mean age of the respondents was 35 years. There were significantly higher female participants, 205 (65\%, $\mathrm{P}<0.001)$, in the study compared to the male respondents. Among the female respondents, 43 (21\%) were aged 18-24 years, 140 (68\%) were aged 25-59 years, and 22 (11\%) were aged 60 years and over. Similarly, among the male participants, 19 (17\%) were aged 18-24 years, 74 (68\%) were aged 25-59 years, and 16 (15\%) were aged 60 years and over. Slightly over a half (55\%) of the study participants were in employment while the rest were unemployed. The level of education was also assessed in this population and it was found out that up to $62 \%$ had at most a primary level of education. There were 88 (28\%) and $35(11 \%)$ with secondary and tertiary education respectively. Seventy one percent of the participants in study were married with 62 (19\%) made up of the singles. There were 305 (95\%, 95\% CI: 92-97\%) participants in this study who were aware of the existence of trachoma disease in the area. One hundred and fifty nine (50\%) study participants knew of facilities or organizations in the region offering trachoma eye care services. Sixty six (42\%), 8 (5\%), and 14 (9\%) respondents claimed to know of a public health facility, private facility and an NGO or other organizations offering trachoma eye care services respectively. Other respondents cited a combination of these health facilities and organizations as is evident from Table 1. Only three respondents claimed to know of chemists offering these eye care services. Another 224 (71\%) knew of the trachoma eye care services being offered in the public or private health facilities and in NGO's. The major eye care service mentioned was antibiotic treatment (83\%), while others mentioned a combination of both antibiotic treatment and eye surgery (12.5\%). There were 299 (95\%) respondents who felt they needed trachoma eye care services. Amongst them, 246 (77\%) would prefer to visit a public health facility as opposed to private facilities. Out of this number, 80 (32.5\%), 48
(19.5\%), 22 (8.9\%), and 15 (6.1\%) claimed that they would prefer to visit public health facilities because of affordability/low cost, accessibility/convenience, adequacy/high quality services and because of lack of an alternative respectively. Other respondents cited a combination of reasons as shown in Table 2. Among the seventy-two respondents who would prefer to visit a private health facility, 55 (76.4\%) claimed this would be so because of their adequacy/high quality standards while another 9 (12.5\%) said they would do so because of their accessibility/convenience. Most of the respondents, 188 (59\%), felt that the health facilities or NGOs in the region adequately met their needs of trachoma eye care services while 79 (25\%) were of a contrary opinion. There were 54 (17\%) respondents who reported lack of knowledge on whether or not these health facilities or organizations adequately met their needs of trachoma eye care services.

A majority of the respondents, 198 (62.5\%) reported that they would visit a public health facility as their first resort of help in case they suffered from an eye condition. There were 55 (17.4\%) and 53 (16.7\%) who reported that they would visit a private health facility and those who would opt to treat themselves respectively. A few others would seek advice from community members first while others would first opt to consult community/traditional doctors. Forty (13\%) respondents claimed to subscribe to certain cultural beliefs and practices that made them not seek trachoma eye care services available at the health facilities. A majority of them,

Table 2. Communities' attitudes towards available trachoma eye care services.

Variables, levels $\quad$ Sample sive No. (\%)

Do you think, you as an individual need these trachoma eye care services?

Yes us. no

315

246

Reasons for preferring public health facilities

Affordability/low cost

Accessibility/convenience

Adequacy/high quality standards

Lack of alternative/no choice

Affordability/low cost and accessibility/convenience

Affordability/low cost and adequacy/high quality standards

Accessibility/convenience and adequacy/high quality standards

Accessibility/convenience and lack of alternative/no choice

Affordability/low cost, accessibility/convenience and adequacy/high quality standards

Reasons for preferring private health facilities

Accessibility/convenience

Adequacy/high quality standards

Lack of alternative/no choice

Affordability/low cost and adequacy/high quality standards

Accessibility/convenience and adequacy/high quality standards

In your opinion, do health facilities or organizations in this area adequately meet your needs for trachoma eye care services?

Yes

No

321

Don't know
318

$299(95)$

$246(77)$

$80(32.5)$

48 (19.5)

$22(8.9)$

$15(6.1)$

61 (24.8)

9 (3.7)

$7(2.9)$

3 (1.2)

$1(0.4)$

72

9 (12.5)

55 (76.4)

1 (1.4)

3 (4.2)

4 (5.6)

188 (59)

79 (25)

54 (17) 
$30(75 \%)$ said they believed in the efficacy of herbal medicine while the rest claimed to believe in healing through divine intervention.

\section{Discussion}

Findings from this study showed that a majority (95\%) of the respondents were well aware of trachoma disease and the existence of the eye condition in the area and this may be as a result of the high prevalence of the eye disease in the region. The study respondents were able to differentiate trachoma disease from other eye diseases by correctly describing how the disease manifests itself. Livingston, McCarty and Taylor (1998) found out that amongst the subjects of a study done in Australia, four percent of them could not differentiate trachoma from glaucoma disease. ${ }^{8}$ Therefore, a high level of awareness of trachoma disease by the community in Central division would in effect translate to the community being knowledgeable about the available eye care services provided in the region. One half (159) of the respondents in the study claimed to be aware of health facilities and organizations in Central division that offered trachoma eye care services. Of these, $42 \%$ of them were aware of such public health facilities with lesser proportions of $4 \%$ and $9 \%$ being aware of mission facilities and NGOs or other organizations that offered these eye care services respectively. Awareness of trachoma eye care services offered in public facilities was higher partly because health care services offered in government facilities were more known to this community as they are the preferred facilities in the area whenever there is need to seek any form of health care and also given the perception of affordable health care services. Amongst those aware of public health facilities (42\%), this was a low proportion compared to a study done in a rural community in South Africa where it was found that more than three-quarters (78.5\%) of the study participants were aware of eye care services offered in government hospitals. ${ }^{9}$ In a similar study done in Nairobi, most respondents knew of government or public facilities offering various health care services but were not aware that eye care services were also available at these facilities. ${ }^{10}$ Close to three-quarters (71\%) of the study respondents were aware of specific trachoma eye care services being offered in the public or private health facilities or by NGO's in the area. The higher proportion of those who were knowledgeable of antibiotic treatment can be explained by the fact that a lot of awareness is created during the multidrug administration (MDA) programs and campaigns as opposed to eye surgical services.
In a study on utilization and barriers to eye surgical services in rural South India, the reason for under-utilization of eye care services among the rural population was lack of awareness of the existing free-of-cost services offered by non-governmental organizations and low-cost eye surgical services. ${ }^{11}$ There were 299 (95\%) respondents who felt they needed trachoma eye care services and this indicated a positive attitude by the community towards the eye care services available in the region. Expression of need of the available health care services offered in a given area often leads to increased utilization of these services. Similarly, in a study done in rural South India, it was found that close to three quarters $(72.7 \%)$ of the study population admitted to requiring eye care services against visual impairment and ocular diseases. ${ }^{12}$ Contrary to the study findings on the communities' positive attitudes regarding their need for eye care services, it was reported that attitudinal reasons like felt there was no need for eye care services were cited by a greater proportion of study participants in Timor-Leste. ${ }^{13}$ Slightly more than a half of the respondents (59\%) felt that the health facilities or NGOs in the area adequately met their needs of trachoma eye care. This meant that a significant proportion (40\%) of the respondents interviewed were not satisfied by what was being done by health facilities and NGOs towards trachoma eye care and felt more needed to be done. This proportion was similar to that of a study done in South Africa where it was reported that only $59.3 \%$ of the respondents were satisfied with the eye care services received at the hospitals. ${ }^{9}$ Seventy seven (77\%) percent of the respondents preferred to visit a public health facility as opposed to visiting private facilities, it was observed that a majority (32.5\%) preferred the public health facilities claiming affordability of the services provided. A lesser proportion (8.9\%) preferred public health facilities because of their adequacy and quality of the services. This contrasts a study done in Limpopo province, South Africa where a bigger proportion (68.3\%) claimed that government hospitals offered good quality eye care services. ${ }^{9}$ In another study in Fiji, of the study participants who sought eye care, majority $(75.6 \%)$ of them preferred services offered in government facilities. ${ }^{14}$ Amongst the respondents (23\%) who preferred private facilities, most (76.4\%) of them attributed their choice to the adequate and high quality services offered in these facilities. Similarly, in a study done in Timor-Leste, it was reported that satisfaction with treatment from private facilities was higher than that from government service providers. ${ }^{13}$ Another study done in Kibera and Dagoretti divisions of Nairobi reported that the choice of private health facilities was based on good service and kind work- ers at the facility. ${ }^{10}$ The communities' eye care seeking practices regarding their eye health was assessed by finding out their first choice of place to seek treatment in case they suffered from an eye disease suspicious of trachoma. Majority of the respondents (62.5\%) preferred to visit a public health facility as a first resort of treatment in case they suffered from an eye condition. There were $17.4 \%$ and $16.7 \%$ who preferred visiting private health facilities and those who opted to treat themselves respectively. Similarly, in a study done in Nairobi, reported that a majority (33\%) of study subjects preferred visiting public health facilities when seeking eye care, $21.5 \%$ preferred mission hospitals, while the rest opted for private clinics, optical shops, chemists and traditional healers. ${ }^{10} \mathrm{~A}$ higher preference of eye care services offered in public health facilities might be attributed to the perception the community may have of the affordability of health care services available in these facilities as was reported earlier. But in another study done in Korogosho slums of Nairobi, it was reported that most of the study subjects preferred seeking eye care from mission hospitals, city council clinics and free eye camps. ${ }^{15} \mathrm{~A}$ smaller proportion (3.2\%) would seek treatment from community/traditional doctors as their first resort in case they suffered from an eye condition suspicious of trachoma. Therefore, it can be assumed that the proportion of those who would prefer seeking eye care from community/traditional doctors would be greater in the more remote regions of Kajiado County where conventional health care is relatively inaccessible to the community. In another study it was reported that the majority of subjects with blinding eye problems from rural areas who were seen in orthodox health institutions had first consulted traditional healers. ${ }^{6}$ Rural communities have a strong faith in and respect for traditional healers, who provide the first line of medical attention for most people in remote areas where modern medical services are inaccessible. ${ }^{16}$ But in a study done in Uganda, close to half (44\%) of the respondents in the study used traditional remedies to treat their eye conditions in spite of them living in close proximity to modern health units. ${ }^{17}$ Twenty four respondents of those who admitted to subscribing to cultural beliefs and practices, believed in the efficacy of herbal medicine. Such communities have strong attachments to their cultural beliefs and this norm may even be stronger in communities living in the more remote regions of the county where conventional eye care is unavailable or inaccessible. In agreement with this finding, was a study done in South Africa, which found that five percent of the study population consulted traditional healers for certain eye problems rather than seeking eye care from the government hospitals even though, the public eye 
care services were readily available and affordable. Some of the factors that influenced the use of eye care services in a rural community included traditional and personal beliefs about the western type of eye care services. ${ }^{18} \mathrm{~A}$ further (24\%) of those who subscribed to cultural beliefs and practices claimed to believe that healing from any health condition only came from a higher spiritual power. Similarly, in a study in Nigeria, a few of the study participants with eye problems claimed to have stopped going to the hospital because their condition became worse. As a result, they relied on spiritual care, self-medication and use of local herbal medicine. ${ }^{6}$

\section{Conclusions}

In order to decrease the debilitating effects of trachoma disease in the community, this study intended to bridge a gap of knowledge by providing in depth information of whether the community was knowledgeable of the available eye care services, their perception and attitudes towards these services and their eye health seeking behavior relative to the available eye care services. This information would then be pertinent towards prevention and control of the eye condition and eventually bringing down its prevalence towards meeting the Vision 2020 goal of eliminating the disease which has been a neglected tropical disease of public health concern. Therefore, the study concluded that level of awareness of trachoma disease in Central Division of Kajiado County was significantly high. Close to three quarters of the respondents were knowledgeable about the available trachoma eye care services in the area and slightly more than half of the respondents were knowledgeable about the health facilities providing these eye care services in the region.

The community in Central Division had a positive attitude towards the available trachoma eye care services in the area, with most of them admitting need for these services. Public health facilities were the preferred first resort of treatment by most of the community members in the area. There is need for rigorous awareness campaigns on the available trachoma eye care services, especially amongst those living in the more remote and hard to reach areas of Kajiado County. Positive atti- tudes towards the services need to be reinforced through community-based educational programs focused on strategies for overcoming personal obstacles to eye care.

\section{Limitations}

The study was carried out amongst the community members living in Central division, who are relatively closer to the health facilities. Therefore, the results from this study cannot be generalized to those living in the more remote areas of the County. This is because, based on the findings of the current study we would expect varied levels of knowledge, attitudes and eye care seeking practices because of the unavailability of trachoma eye care services in these hard-to-reach areas.

\section{References}

1. World Health Organization. Neglected Tropical Diseases:World Health Organization; 2014. Available from: http://www.who.int/neglected_diseases/ab out/en/ Accessed: January 2014.

2. ICTC. The End In (Of) Sight: 2020 INSight. International Coalition for Trachoma Control; 2011.

3. Beatty W, Bryne G, Morrison R. Repeated and persistent infection with chlamydia and the development of chronic inflammation and disease. Trends Microbiol 1994;2:94-8.

4. World Health Organization. Trachoma control: a guide for program managers. Geneva: World Health Organization; 2006.

5. Bhagwan J, Rastogi I, Malik J, Dhull C. Knowledge, attitude and practices regarding cataract surgery among severe cataract cases in Hanyana. Indian J Comm Med 2006;31:66-8.

6. Ashaye A, Ajuwon A, Adeoti C. Perceptions of blindness and blinding conditions in rural communities. J Nat Med Assoc 2006;98:887-93.

7. Mugenda 0, Mugenda A. Research methods: quantitative and qualitative approaches. Nairobi: African Centre for Technology Studies (ACT); 2003.

8. Livingston PM, McCarty CA, Taylor HR. Knowledge, attitudes, and self care prac- tices associated with age related eye disease in Australia. $\mathrm{Br} \mathrm{J}$ Ophthalmol 1998;82:780-5.

9. Ntsoane MD, Oduntan OA, Mpolokeng BL. Utilisation of public eye care services by the rural community residents in the Capricorn district, Limpopo Province, South Africa. Afr J Prim Health Care Fam Med 2012;4:7.

10. Kimani K, Karimurio J, Gichuhi S, et al. Barriers to utilization of eye care services in Kibera and Dagoreti, Kenya. East Afr J Ophthalmol 2008;82:506-8.

11. Chandrashekhar TS, Bhat HV, Pai RP, Nair SK. Coverage, utilization and barriers to cataract surgical services in rural South India: results from a population-based study. Public Health 2007;121:130-6.

12. Nirmalan PK, Katz J, Robin AL, et al. Utilisation of eye care services in rural south India: the Aravind comprehensive eye survey. Br J Ophthalmol 2004;88:123741.

13. Palagyi A, Ramke J, Du Toit R, Brian G. Eye care in Timor-Leste: a population-based study of utilization and barriers. Clin Exp Ophthalmol 2008;36:47-53.

14. Brian G, Maher L, Ramke J, Palagyi A. Eye care in Fiji: a population-based study of use and barriers. Ophthalmic Epidemiol 2012;19:43-51.

15. Nyaga G, Kollmann M, Kimani K, Ilako D. Magnitude and pattern of eye diseases in Korogocho slum, Nairobi. East Afr Ophthalmol J 2007;13:29.

16. Chana H, Schwab L, Foster A. With an eye to good practice: traditional healers in rural communities. World Health Forum 1994;15:144-6.

17. Nyathirombo A, Mwesigye F, Mwaka A. Traditional eye health practices in Atyak Sub-county, Nebbi district, Uganda. JOECSA 2013;16.

18. Oduntan A, Raliavhegwa M. An evaluation of the impact of the eye care services delivered to the rural communities in the Mankweng Health sub-district of the Northern Province. Afr Optom 2001;60:716. 